\title{
Entrenamiento de voluntarios en Primera Ayuda Psicológica y estrategias de afrontamiento
}

\section{Training of Volunteers in Psychological First Aid and Coping Strategies}

\author{
Luciana Becerra ${ }^{1}$ \\ https://orcid.org/0000-0002-7173-404X \\ Vera Bail Pupko \\ https://orcid.org/0000-0003-4389-959X \\ Pablo Depaula ${ }^{3}$ \\ https://orcid.org/0000-0002-5610-9131 \\ Susana Azzollini ${ }^{4}$ \\ https://orcid.org/0000-0002-3192-5087
}

${ }_{1,2,3,4}$ Centro de Investigaciones Sociales y Humanas para la Defensa, Universidad de la Defensa Nacional, Argentina
${ }_{3,4}$ Consejo Nacional de Investigaciones Científicas y Técnicas, Argentina

Resumen. Objetivo. Analizar la eficacia del personal voluntario y profesional que asiste en emergencias (Bomberos Voluntarios, Defensa Civil, Cruz Roja) en un programa de entrenamiento en la Primera Ayuda Psicológica y su relación con indicadores de empatía y estrategias de afrontamiento. Método. Se realizó un estudio cuantitativo, comparativo correlacional, con una muestra de 198 voluntarios que pertenecen a instituciones que intervienen en emergencias. Se utilizó un programa de simulación sobre respuestas a víctimas de catástrofes (Sistema Interactivo de Primera Ayuda Psicológica y el Inventario de Valoración y Afrontamiento). Resultados. Los voluntarios que expresan que manejan mejor una situación estresante lograban un rendimiento más eficaz en el programa de simulación. La valoración del contexto como amenazante o indiferente, perjudicaba la ejecución del protocolo de PAP propuesto en la ejercitación mediante software.

Palabras clave. Primera Ayuda Psicológica, voluntarios, empatía, estrategias de afrontamiento.

Abstract. Objective. To analyze the effectiveness of volunteer and professional personnel who assist in emergencies (Volunteer Firefighters, Civil Defense, Red Cross) in a Psychological First Aid training program and its relationship with indicators of empathy and coping strategies. Method. A quantitative, comparative, and co-relational study was carried out with a sample of 198 volunteers belonging to institutions that intervene in emergencies. A simulation program on responses to victims of catastrophes was used, Interactive System in Psychological First Aid and the Coping Strategies Inventory. Results. Volunteers, who state that handle better stressful situations, achieve a more effective performance in the simulation program. The assessment of the context as threatening or indifferent impaired the execution of the proposed PFA protocol using software.

Keywords. Psychological First Aid, Volunteers, Efficacy, Empathy, Coping Strategies.

${ }^{1}$ Luciana Becerra. Centro de Investigaciones Sociales y Humanas para la Defensa, Universidad de la Defensa Nacional, Argentina. Dirección postal: Aviadora Lorenzini 2419, El Palomar, Buenos Aires, Argentina (CP 1684). E-mail: becerraluciana1@gmail.com ${ }^{2}$ Vera Bail Pupko. Centro de Investigaciones Sociales y Humanas para la Defensa, Universidad de la Defensa Nacional, Argentina. Email: verabailpupko@gmail.com

${ }^{3}$ Pablo Depaula. Centro de Investigaciones Sociales y Humanas para la Defensa, Universidad de la Defensa Nacional, Argentina. Consejo Nacional de Investigaciones Científicas y Técnicas, Argentina. Email: lic_pablodepaula@yahoo.com.ar

${ }^{4}$ Susana Azzollini. Centro de Investigaciones Sociales y Humanas para la Defensa, Universidad de la Defensa Nacional, Argentina. Consejo Nacional de Investigaciones Científicas y Técnicas, Argentina. Email: susana1060@yahoo.com.ar

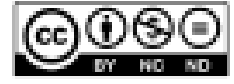

Esta obra está bajo una licencia de Creative Commons Reconocimiento-NoComercial-SinObraDerivada 4.0 Internacional. 


\section{Introducción}

La Primera Ayuda Psicológica (PAP) se implementa en la atención de víctimas en situaciones de catástrofes y emergencias para favorecer la recuperación de la comunidad (Organización Mundial de la Salud [OMS] et al., 2012). Según El Proyecto Esfera (2011) y el Comité Permanente entre Organismos (Inter-Agency Standing Commitee [IASC], 2007), la PAP describe una respuesta humana, de apoyo a otro ser humano que está sufriendo y que puede necesitar ayuda.

Además de los modelos de PAP dirigidos a desplegar acciones psicosociales preventivas (OMS et al., 2012; IASC, 2007), de acuerdo con Roberts et al. (2019), en los últimos años se ha incrementado la aplicación de modelos de intervención del TEPT (Trastorno de Estrés Postraumático - Kearns et al., 2012; Qi et al., 2016), basados en intervenciones de sesiones múltiples dirigidas a cualquier individuo expuesto a un evento traumático con el objetivo de prevenir el desarrollo del TEPT, intervenciones dirigidas a personas con un factor de riesgo específico conocido o sospechado, e intervenciones dirigidas a personas que son claramente sintomáticas. En relación con la muestra bajo estudio en el presente trabajo, son dables de considerar las intervenciones rápidas ante reacciones de estrés agudo en ocupaciones de alto riesgo (militares, Svetlitzky et al., 2019, migrantes, Levy et al., 2020, etc.).

Con el objetivo de analizar la eficacia de las intervenciones psicológicas enfocadas en la prevención del TEPT, recientemente, Roberts et al. (2019) realizaron un estudio de revisión sistemática basándose en diversas búsquedas en las bases de datos del Cochrane Central Register of Controlled Trials (CENTRAL), MEDLINE, Embase, PsycINFO y ProQuest's, y en la Published International Literature On Traumatic Stress (PILOTS) publicados hasta el día 3 de marzo del año 2018. Además, se realizó una búsqueda previa en el CENTRAL y en las bases de Ovid a través del Cochrane Common Mental Disorders Controlled Trial Register (CCMD-CTR) de la literatura publicada hasta el mes de mayo del año 2016. Por último, se realizaron búsquedas manuales en listas de referencias de guías relevantes, revisiones sistemáticas e informes de estudios (los que compartieron con expertos de gran jerarquía en el campo psicológico o psicoterapéutico en cuestión). De esta manera, se concluye que los primeros auxilios psicológicos se han prescrito, con mayor frecuencia, como una forma inicial de intervención sustentada en este tipo de modelo o paradigma (National Child Traumatic Stress Network \& National Center for PTSD, [NCTSN/NCP], 2006), también, que la prevención del TEPT a través del accionar psicológico o psicoterapéutico precoz fomenta estrategias de manejo del estrés y existen diversos estudios experimentales que así lo consideran (Biggs et al., 2016; Wijesinghe et al., 2015).

Desde el equipo de investigación, se toma el modelo de intervención desarrollado por Farchi et al (2018) que enfoca la Primera Ayuda Psicológica desde una intervención cognitivofuncional inmediata. El modelo busca estandarizar las acciones de socorristas en situaciones de emergencia o catástrofe para las personas que presentan una reacción de estrés agudo. Tiene la intención de ayudar a que la persona pase de la impotencia y la pasividad a un funcionamiento activo y efectivo inmediatamente después de percibir un evento como traumático. El modelo se basa en cuatro ejes: (a) Resistencia, (b) Sentido de coherencia, (c) Autoeficacia y (d) en la Neuropsicología de la respuesta al estrés, el cual se centra en lograr en las personas el tránsito de una hiperactividad del sistema límbico a una activación de la corteza prefrontal durante eventos estresantes. Este modelo de intervención se aparta de otros modelos más generales y orientados a la expresión narrativa y la empatía, ha sido reconocido por el Ministerio de Salud de Israel como el modelo nacional de primeros auxilios psicológicos, y ha sido trasladado a capacitaciones en distintos países, incluyendo Argentina. 
Palacios y Téllez (2017) analizaron la reacción de la población vulnerable ante un terremoto (el sismo ocurrido en México durante el año 2017). Se sospechaba, como en muchas otras poblaciones vulnerables, que el desastre iba a potenciar el desastre social ya percibido e incrementar el riesgo de desajuste individual y colectivo. Sin embargo, de forma inmediata la ciudadanía inició y potenció, con el uso de las tecnologías de la información y comunicación, acciones de organización informal con el objetivo de contener el daño y atender las necesidades más apremiantes de los damnificados. Se convocaron voluntarios médicos, enfermeras, psicólogos, rescatistas, etc. para formar grupos de intervención ante la situación de emergencia. El objetivo de la intervención se centró en tres aspectos: atender el malestar emocional resultado de la experiencia traumática, restablecer los recursos individuales cognitivos y emocionales ante la crisis (reconocimiento de la situación, del riesgo presente, toma de decisiones de autocuidado y seguridad personal y familiar) y la prevención del desarrollo de conductas psicopatológicas que debilitaran la salud de los individuos y afectaran su capacidad de respuesta posterior.

El aspecto preventivo es importante porque las catástrofes socio-naturales constituyen fenómenos sociales que se caracterizan por afectar disruptivamente a las comunidades en donde se ocasionan, provocando en los afectados diversos daños psíquicos, vinculares, sociales, económicos, institucionales, entre otros, alcanzando también a sus grupos de pertenencia y referencia (Ascaini et al., 2016). Para estos autores, que los acontecimientos sean o no traumáticos dependerá de las capacidades de los sujetos afectados, de sus historias de vida, de la intensidad de los eventos y de la posibilidad de elaboración colectiva.

Colussi (2017) remarca que los terremotos, inundaciones o eventos similares vienen a evidenciar situaciones de exclusión social y desarrollo desigual que exponen a estados de alta vulnerabilidad a enormes grupos humanos. Muchos de los daños ocasionados por estos fenómenos podrían tener impactos menores a los que alcanzan, o simplemente podrían no ocasionarse, si las sociedades afectadas presentaran otros patrones de organización. En la actualidad, dado el escaso grado de desarrollo de nuestros conocimientos científicos, muchos factores de peligro no se pueden prever, y menos aún eliminar. Pero sí puede evaluarse el grado de vulnerabilidad de un grupo humano y, consecuentemente mitigarse, con lo que se reduciría el impacto negativo de un desastre.

El entrenamiento de socorristas y de la comunidad general en PAP incrementa la iniciativa, la confianza y la probabilidad de brindar ayuda por parte de los miembros de la comunidad al momento de la emergencia (Bail et al., 2017; Herrman, 2012; Sun \& Wallis, 2011).

Plummer et al. (2009) destacan que la PAP constituye el modelo de abordaje en crisis más reciente y eficaz, enfoque que intenta generar un alivio precoz en las víctimas al combinar los componentes más efectivos de los modelos precedentes y eliminando los más inefectivos y dañinos (Everly \& Flynn, 2005). Sin embargo, un estudio de revisión sistemática en el que se analizó bibliografía indexada hasta el mes de julio del año 2013 en cinco bases de datos (MEDLINE, PsycINFO, The Cochrane Library, PILOTS and G-I-N) evidenció la escasa evidencia de la efectividad de las intervenciones en PAP sugiriendo incrementar los estudios experimentales y observacionales sobre dicha técnica (Dieltjens et al., 2014).

Recientemente, Kureckova et al. (2017) han argumentado que el aspecto central del entrenamiento en primeros auxilios psicológicos basado en la experiencia consiste en trabajar sobre situaciones simuladas, mediante el análisis detallado el desempeño, la eficacia y los procesos emocionales desplegados por los sujetos entrenados, ayudándolos 
a manejar las situaciones de emergencia de manera efectiva y, sobre todo, disminuyendo las reacciones emocionales inadecuadas. Bajo este panorama, algunos autores (Hambrick et al., 2014) sugieren que al aplicar un programa de entrenamiento, un elevado nivel de conciencia (awareness) presente en los voluntarios al momento de utilizar la PAP, las propias percepciones del uso de la técnica junto al monitoreo metacognitivo de las experiencias previas asociadas, sumados a un concomitante sentimiento de confianza en la aplicabilidad de la misma como respuesta al evento desastroso, podrían asegurar un alto nivel de efectividad del dispositivo aplicado.

La activación cognitiva que estimula el trabajo de la corteza prefrontal a la que aspira la PAP a los fines de reducir precozmente la hiperactivación emocional (amigdalina) de las víctimas (Farchi \& Hantman, 2013), requeriría de los voluntarios la posesión de dominios cognitivos principalmente (uso del análisis deliberado y de la creatividad), dominios psicomotores (habilidades comunicacionales -verbales y no verbales- para facilitar la interacción social) y, en último lugar, dominios afectivos (expresión actitudinal de emociones, sentimientos, valores y motivaciones; McCabe et al., 2014).

Al considerar el entrenamiento y la eficacia de los voluntarios en PAP, De Leo et al. (2003) sugieren el desarrollo de sistemas de realidad virtual (simulaciones audiovisuales) para la evaluación de las decisiones y procedimientos adoptados correctamente ante la gran incertidumbre ambiental y operacional propia de las situaciones de emergencia ( $\mathrm{Ha}$ et al., 2015) mejorando la toma de decisiones (Hogarth \& Soyer, 2015). La preparación a través del entrenamiento especializado de los voluntarios y la confianza en las propias competencias y conocimientos parecen impactar positivamente sobre el nivel de bienestar posterior al evento desastroso; mientras que el sentimiento de no hallarse debidamente entrenado (percepción de maestría profesional y/o capacidades de equipo) se asocia a altos niveles de distrés (Brooks et al., 2016). El hecho de proporcionar capacitación en primeros auxilios psicológicos a los rescatistas provocaría también una mayor propensión de estos individuos a aplicar dichas habilidades sobre la comunidad en general. Esto es parte importante de su fuerza de trabajo (Crawford et al., 2015; Reifels et al., 2014) y se incrementa a su vez sus niveles de resiliencia y autoeficacia (Farchi et al., 2014).

En Argentina, los Bomberos Voluntarios, el personal de Defensa Civil y Cruz Roja son los primeros en asistir a los afectados en las zonas de emergencia y catástrofe. A partir de los contactos institucionales iniciales de la investigación, se informa al equipo de investigación que ha desarrollado el presente estudio, que su entrenamiento no incluye, hasta el momento, capacitación en PAP. Brindarles esta capacitación, puede optimizar su desempeño y además plantea el desafío de pensar cuáles serían las características deseables en un individuo que reciba entrenamiento en PAP, para que este resulte más efectivo.

El proyecto de investigación, del cual este trabajo es parte, buscaba identificar las variables psicológicas que podían favorecer o dificultar el entrenamiento mediante simulación de PAP, bajo el supuesto que dichas dificultades o facilidades tenderían a replicarse en la aplicación en situaciones de catástrofe.

En un estudio anterior (Azzollini et al., 2017) se abordó en una muestra similar, pero integrada por una menor cantidad de participantes, la relación entre la eficacia alcanzada en un programa de simulación de PAP e indicadores de empatía. Se concluye que esta variable resulta relevante, pero su influencia sobre el aprendizaje del protocolo propuesto es indirecta, por tanto es necesario profundizar sobre otros factores que intervienen en el 
entrenamiento. Con base en esto, se decidió evaluar las estrategias de afrontamiento al estrés en relación con la eficacia en el entrenamiento en PAP.

El afrontamiento, vinculado al manejo de emergencia y estrés, se define como "el cambio cognitivo o conductual constante para manejar demandas internas o externas específicas que se juzgan muy exigentes o que exceden los recursos de la persona" (Navarro, 2004, p. 172).

Con respecto al entrenamiento en PAP, se utilizó el programa de simulación denominado Software Interactivo de Primera Ayuda Psicológica (Azzollini et al., 2015) para el análisis de la eficacia en el entrenamiento. Este sistema es producto de investigaciones llevadas a cabo en el Centro de Investigaciones Sociales y Humanas para la Defensa (CISOHDEF), en el Colegio Militar de la Nación (Facultad del Ejército, Universidad de la Defensa Nacional) y en el Instituto de Investigaciones en Psicología (Universidad de Buenos Aires). Se desarrolló un protocolo basado en las pautas de la guía de PAP de la Organización Mundial de la Salud et al. (2012) y en la adaptación a nuestro medio del modelo de las "6 C" propuesto por Farchi et al., (2014), y adaptado en Argentina como el modelo de las "4 C" (Azzollini et al., 2017). Dicho protocolo consta de 10 acciones sencillas, focalizadas en la comunicación cognitiva, el restablecimiento de la sensación de control, el compromiso y la continuidad.

El objetivo del estudio fue analizar el rendimiento de Bomberos Voluntarios, personal de la Cruz Roja y Defensa Civil en un programa de simulación de PAP y la relación de dicho desempeño con indicadores de estrategias de afrontamiento.

\section{Método}

Se realizó un estudio cuantitativo, no experimental de alcance descriptivo, comparativo y correlacional, de corte transversal con un muestreo intencional simple. La muestra estuvo compuesta por 198 voluntarios de Bomberos Voluntarios, Cruz Roja Argentina y de Defensa Civil. Todos los sujetos participaron de una capacitación previa para actuar en PAP basada en el modelo de las 4 C. Dicha capacitación constaba de un encuentro donde se exponían las bases del modelo de las 4 C, se especificaban las 10 acciones sistematizadas por el protocolo, se ejemplificaba en juegos de rol y se despejaban las dudas sobre los temas expuestos y el protocolo de acción propuesto. En todos los casos, la capacitación fue llevada a cabo por investigadores formados en PAP que pertenecen al equipo de investigación. Transcurrida una semana de la capacitación, se convocaba a todos los participantes nuevamente para responder los cuestionarios referidos a las variables de empatía y estrategias de afrontamiento y completar el programa de entrenamiento y simulación Sistema Interactivo de Primera Ayuda Psicológica (SIPAPSI).

Cabe señalar que todos los participantes colaboraron de forma gratuita y voluntaria. Mediante el acuerdo con las instituciones a las que pertenecían se consensuó el plan de acción, la capacitación y se explicitaron las condiciones de participación. Los encuentros se realizaron siempre en las sedes institucionales a las que pertenecían los voluntarios. Se resguardó la confidencialidad y se hicieron las devoluciones solicitadas sobre los resultados de la investigación. Los participantes fueron informados de las condiciones y características del estudio, pudiendo abandonar el mismo en cualquier momento. El consentimiento informado fue brindado en la toma de datos posterior a la capacitación.

Debido a los tiempos dispuestos por las instituciones participantes y las características del programa de simulación, por tanto, no se realizaron medidas basales de los grupos. Se descartaron los protocolos incompletos y los que pertenecían a voluntarios que no habían participado de la capacitación. 
Instrumentos de recolección de datos

Para evaluar si el protocolo expuesto en la capacitación era replicado en una simulación, se utilizó el programa SIPAPSI desarrollado por el equipo de investigación, (Azzollini et al., 2015). En este software se presentan 10 series de 4 videos cada una con acciones posibles de asistencia a víctimas de emergencia. En cada una de las series, hay solo uno de los videos que se corresponde con las pautas de acción efectiva propias del protocolo de PAP propuesto. Inicialmente, se presenta un reporte o noticia periodística que informa acerca de la naturaleza del evento sobre el cual los usuarios deben aplicar la PAP a una de sus víctimas, por ejemplo, en un accidente de tránsito ferroviario con múltiples víctimas (ver Figura 1 y Figura 2).

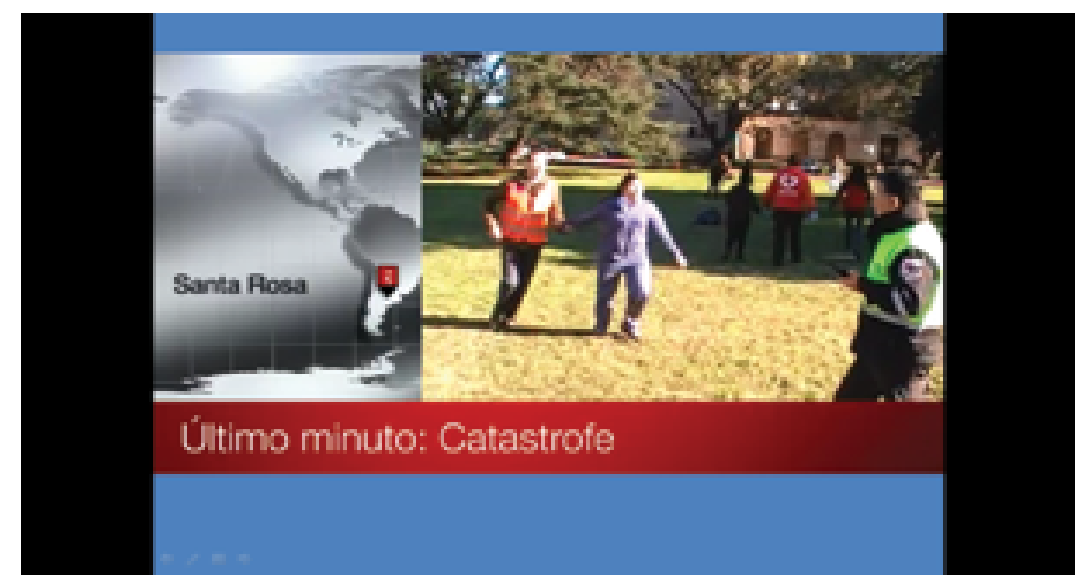

Figura 1. Placa de presentación de la catástrofe. La pantalla de inicio del SIPAPSI muestra a los sujetos evaluados un reporte periodístico con escenas de la catástrofe en la que deberán actuar asistiendo a una de las víctimas del evento.

\section{¿Yo qué haría?}
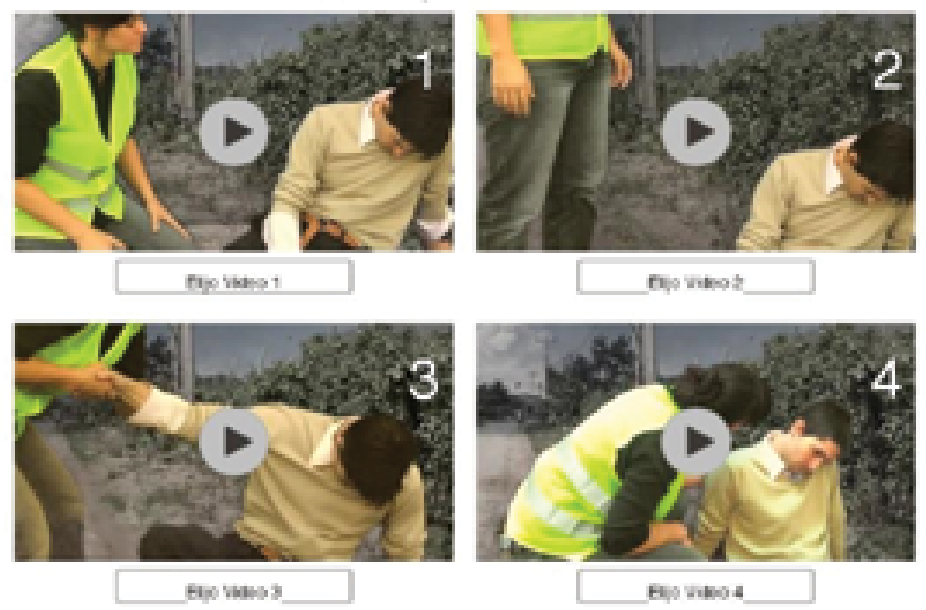

Figura 2. Placa de presentación de la catástrofe. La pantalla de inicio del SIPAPSI muestra a los sujetos evaluados un reporte periodístico con escenas de la catástrofe en la que deberán actuar asistiendo a una de las víctimas del evento. 
La variable eficacia del entrenamiento en PAP es operacionalizada como la cantidad de respuestas correctas alcanzadas al momento de operar el programa SIPAPSI. La respuesta es correcta cuando el sujeto elige la opción del video que se adecúa al protocolo presentado en las capacitaciones, que concuerda con el modelo desarrollado. La operacionalización de la variable, entonces oscila entre valores de 0 y 10 puntos, considerándose 10 el indicador de mayor eficacia del entrenamiento. Cabe aclarar que, en este trabajo la eficacia es entendida en relación con el aprendizaje del modelo propuesto, sin llegar a evaluar su ejecución real en situación de catástrofe o emergencia.

Los sujetos evaluados respondieron individualmente a la prueba a través de ordenadores y auriculares, en la cual debían seleccionar con el puntero de un mouse la opción de video de cada una de las 10 series que consideraba en la acción de PAP correcta.

Para la evaluación de los indicadores de valoración y estrategias de afrontamiento al estrés se utilizaron los datos obtenidos del Inventario de Valoración y Afrontamiento (IVA) de Cano-Vindel y Miguel-Tobal (1999), validado por Leibovich de Figueroa (1999). El inventario consta de 42 ítems con opciones de respuesta en una escala likert que oscila entre 1 y 5 puntos. La consigna de la escala fue adaptada al estudio y se planteó una situación de catástrofe o emergencia como punto de referencia para la respuesta de los enunciados. Las respuestas a los reactivos se promedian, agrupándose en 9 escalas, de las cuales las primeras 3 refieren a la valoración de la situación y las siguientes 6 a los estilos de afrontamiento. Un puntaje promedio más cercano a 5 se interpreta como una identificación más fuerte con el estilo de afrontamiento o una utilización más frecuente de este.

Los tres modos de valoración de la situación que operacionaliza el instrumento son:

-VSA: Valoración de la Situación como Amenazante.

-VSD: Valoración de la Situación como Desafío.

-VSI: Valoración de la Situación como Indiferente.

Las 6 estrategias de afrontamiento ante la situación de emergencia que evalúa son:

-ACS: Afrontamiento Cognitivo dirigido a cambiar la Situación.

-ACE: Afrontamiento Cognitivo dirigido a reducir la Emoción.

-ACMS: Afrontamiento Conductual Motor dirigido a cambiar la Situación.

-ACME: Afrontamiento Conductual Motor dirigido a reducir la Emoción.

-AP: Afrontamiento Pasivo.

-AE: Afrontamiento Evitativo.

El inventario de estrategias de afrontamiento cuenta con estudios previos de validez y confiabilidad que avalaban la interpretación de los resultados obtenidos mediante su uso (Leibovich de Figueroa, 1999).

\section{Descripción de la muestra}

Todos los sujetos recibieron la capacitación sistematizada a través de las investigaciones mediante profesionales entrenados en el protocolo de PAP desarrollado. Tanto las capacitaciones como la ejecución del SIPAPSI se realizaron mediante acuerdos institucionales en las sedes de reunión y trabajo de Bomberos Voluntarios y Defensa Civil. 
La participación fue voluntaria y se aseguró la confidencialidad de las respuestas. Las condiciones de la evaluación, así como las características de la actividad fueron explicitadas previamente y se obtuvo el consentimiento informado de todos los participantes.

Participaron del estudio un total de 198 sujetos: 139 Bomberos Voluntarios de distintas secciones (Berazategui, San Martín, Sarandí, La Boca, Lanús, Ituzaingó), 42 voluntarios de la Cruz Roja Argentina y 17 voluntarios de Defensa Civil, todos residentes en la Ciudad de Buenos Aires y en el Conurbano Bonaerense. Las edades oscilaban entre los 14 y 67 años $(M=32.9 ; D T=10.7)$. De los voluntarios, 3 jóvenes menores de 18 años pertenecían a Cruz Roja Argentina, institución que permite participar en capacitaciones y actividades de voluntariado a partir de los 14 años. Respecto al género la muestra se distribuyó en un $38.9 \%$ de mujeres y un $61.1 \%$ de varones.

Consultados sobre su experiencia previa como voluntarios en situaciones de catástrofe, el $50.5 \%$ ya había participado en situaciones de emergencia comunitaria en actividades de rescate o asistencia a víctimas. Asimismo, el $41.9 \%$ admitió haber padecido situaciones de catástrofe por fuera de su rol de rescatistas.

Los participantes respondieron al programa SIPAPSI y a varios inventarios, siendo el IVA uno de ellos. Se ha publicado en otros trabajos información parcial sobre los resultados que consideran distintas composiciones muestrales (Grinhaus et al., 2020; Azzara et al., 2019; Azzollini et al., 2018, 2017; Bail et al., 2017) conforme al avance en el acuerdo con las instituciones y progreso del proyecto de investigación que contextualizó las actividades o trabajos de campo. El presente reporte se focaliza en el análisis de la relación entre el rendimiento alcanzado en el SIPAPSI y los indicadores de estrategias de afrontamiento.

\section{Resultados}

Se analizaron los datos mediante el paquete estadístico IBM SPSS versión 22.0 para Windows. Se calcularon los valores muestrales para los datos recopilados a través del IVA, así como para la cantidad de respuestas correctas arrojadas por el SIPAPSI. Debido a la distribución de las variables en la muestra, se utilizaron para la comparación de grupos y el análisis de las correlaciones, estadísticos no paramétricos. Para la comparación de dos grupos se utilizó $U$ de Mann Whitney $(U), \mathrm{H}$ de Kruskall Wallis $(H)$ para verificar la diferencia entre más de dos grupos y la Rho de Spearman $\left(r_{s}\right)$ para correlaciones de variables escalares. En aquellos casos que corresponda, se informa a modo descriptivo los Rango Promedio $\left(R_{\chi}\right)$ para cada grupo.

Los resultados son organizados en tres apartados. En el primero se describen los puntajes de la escala IVA y la correlación entre dichos puntajes, la edad, el género y la experiencia previa en emergencias. En la segunda sección, se presentan los indicadores de eficacia en el entrenamiento en PAP y las asociaciones halladas a partir de los datos relevados en cuanto a los datos demográficos y la experiencia previa. En la tercera y última sección, se analiza la interrelación entre los indicadores de estrategias de afrontamiento y desempeño en el programa SIPAPSI.

\section{Valoración y estrategias de afrontamiento}

La Tabla 1 muestra los valores descriptivos obtenidos en la escala de Valoración y Afrontamiento ante el estrés. En relación con el modo de valoración de las situaciones de emergencia o catástrofe, se observa una mayor tendencia a identificar dichos contextos como desafío. En cuanto a los modos de afrontamiento, los voluntarios identifican con mayor frecuencia estrategias para reducir la emoción (tanto cognitivas como motoras) y conductas dirigidas a cambiar la situación. 
Tabla 1

Descripción de los puntajes del IVA en toda la muestra $(N=198)$

\begin{tabular}{lccc}
\multicolumn{1}{c}{ Escala } & Mdn. & Min. & Máx. \\
\hline Valoración de la Situación como Amenazante (VSA) & 2.3 & 0.3 & 4.3 \\
Valoración de la Situación como Desafío (VSD) & 3.3 & 1.0 & 5 \\
Valoración de la Situación como Indiferente(VSI) & 1.6 & 0.0 & 4.6 \\
Afrontamiento Cognitivo dirigido a cambiar la Situación (ACS) & 3.1 & 0.6 & 5 \\
Afrontamiento Cognitivo dirigido a reducir la Emoción (ACE) & 3.6 & 1.0 & 5 \\
Afrontamiento Conductual Motor dirigido a cambiar la Situación (ACMS) & 3.6 & 1.5 & 5 \\
Afrontamiento Conductual & 3.5 & 0.6 & 5 \\
Motor dirigido a reducir la Emoción (ACME) & & & \\
Afrontamiento Pasivo (AP) & 2.0 & 0.0 & 5 \\
Afrontamiento Evitativo (AE) & 1.0 & 0.0 & 5 \\
\hline
\end{tabular}

La comparación de los puntajes en la escala IVA en relación con el género, mostró diferencias entre voluntarios hombres y mujeres en la escala $\mathrm{ACME}\left(p<.05 ; R_{1}\right.$ mujeres $=$ $106.5 ; R_{2}$ hombres $\left.=95.0\right)$ y AP $\left(p<.01 ; R_{1}\right.$ mujeres $=108.0 ; R_{2}$ hombres $\left.=94.0\right)$, obteniendo valores más elevados en ambas escalas en las mujeres.

La edad mostró una leve asociación con el Afrontamiento Cognitivo dirigido a cambiar la Situación (ACS), en la cual se obtuvo una $r_{s}=.172 ; p<.05$, lo cual indicaría que los voluntarios de mayor edad tienden a intentar pensar en cómo cambiar la situación más que los jóvenes. En lo que respecta al tipo de institución, se observaron diferencias estadísticamente significativas en todas las estrategias de afrontamiento analizadas (ver la Tabla 2).

Los Bomberos Voluntarios presentan valores más bajos en todas las dimensiones, mientras que los de Defensa Civil puntúan más alto, a excepción de los puntajes en las escalas de Valoración de la Situación como Amenazante y Afrontamiento Pasivo, en el que el grupo de Cruz Roja obtiene los mayores valores.

Al comparar los grupos entre pares, se observó que los tres grupos presentan perfiles diferenciados en cuanto la valoración y estilo de afrontamiento en la mayoría de las dimensiones. Los bomberos se diferencian de los otros dos grupos en todas las escalas, a excepción del Afrontamiento Cognitivo dirigido a cambiar la Situación (ACS), y el Afrontamiento Pasivo (AP) que presenta diferencias con los participantes de Cruz Roja respecto a la primera escala mencionada y diferencias con los participantes de Defensa Civil en la segunda. Los voluntarios de Defensa Civil muestran diferencias con los de Cruz Roja en dicha escala (ACS) y en la Valoración de la Situación como Indiferente (VSI, ver la Tabla 3).

La comparación de los estilos de afrontamiento entre los voluntarios con y sin experiencias, arrojó valores de $p$ significativos en las escalas Valoración de la Situación como Amenazante $\left(p>.001 ; R_{1}\right.$ con experiencia $=88.80 ; R_{2} \sin$ experiencia $\left.=110.42\right) \mathrm{y}$ Afrontamiento Evitativo $\left(p>.001 ; R_{1}\right.$ con experiencia $=85.71 ; R_{2}$ sin experiencia $\left.=113.58\right)$, además, se presentaron en ambos casos valores más elevados en los voluntarios que no han participado previamente en este tipo de situaciones. 
Tabla 2

Diferencias en escala IVA según tipo de institución

\begin{tabular}{|c|c|c|c|c|}
\hline \multirow[b]{2}{*}{ Escala } & \multicolumn{3}{|c|}{ Rangos promedio } & \multirow{2}{*}{$\begin{array}{c}\text { Valor } \\
\text { de } p \\
H \text { de } \\
\text { Kruskal } \\
\text { Wallis }\end{array}$} \\
\hline & $\begin{array}{c}\text { Bomberos } \\
\text { Voluntarios } \\
(n=139)\end{array}$ & $\begin{array}{c}\text { Cruz } \\
\text { Roja } \\
(n=42)\end{array}$ & $\begin{array}{c}\text { Defensa } \\
\text { Civil } \\
(n=17)\end{array}$ & \\
\hline Valoración de la Situación como Amenazante (VSA) & 86.55 & 135.39 & 116.71 & $.001^{\star \star}$ \\
\hline Valoración de la Situación como Desafío (VSD) & 87.86 & 125.74 & 129.88 & $.001^{\star *}$ \\
\hline Valoración de la Situación como Indiferente(VSI) & 88.97 & 114.17 & 149.35 & $.001^{\star \star}$ \\
\hline $\begin{array}{l}\text { Afrontamiento Cognitivo dirigido a cambiar la } \\
\text { Situación (ACS) }\end{array}$ & 93.66 & 101.07 & 143.38 & $.003^{\star \star}$ \\
\hline $\begin{array}{l}\text { Afrontamiento Cognitivo dirigido a reducir la } \\
\text { Emoción (ACE) }\end{array}$ & 92.37 & 112.37 & 126.00 & $.019^{*}$ \\
\hline $\begin{array}{l}\text { Afrontamiento Conductual Motor dirigido a } \\
\text { cambiar la Situación (ACMS) }\end{array}$ & 90.36 & 118.19 & 128.03 & $.002^{\star \star}$ \\
\hline $\begin{array}{l}\text { Afrontamiento Conductual Motor dirigido a reducir } \\
\text { la Emoción (ACME) }\end{array}$ & 91.70 & 114.37 & 126.56 & $.010^{\star}$ \\
\hline Afrontamiento Pasivo (AP) & 90.39 & 122.18 & 117.97 & $.002^{\star *}$ \\
\hline Afrontamiento Evitativo (AE) & 85.02 & 133.25 & 134.50 & $.001^{\star *}$ \\
\hline
\end{tabular}

${ }^{*} p<.05 .{ }^{* *} p<.01$.

Tabla 3

Diferencias en escala IVA inter-grupos

\begin{tabular}{|c|c|c|c|}
\hline \multirow[b]{2}{*}{ Escala } & \multicolumn{3}{|c|}{ Valor de $p U$ de Mann Whitney } \\
\hline & $\begin{array}{c}\text { Bomberos } \\
\text { Voluntarios } \\
(n=139) / \\
\text { Cruz Roja } \\
(n=42)\end{array}$ & $\begin{array}{c}\text { Bomberos } \\
\text { Voluntarios } \\
(n=139) / \\
\text { Defensa Civil } \\
(n=17)\end{array}$ & $\begin{array}{c}\text { Cruz Roja } \\
(n=42) / \\
\text { Defensa } \\
\text { Civil } \\
(n=17)\end{array}$ \\
\hline Valoración de la Situación como Amenazante (VSA) & $.001^{\star \star}$ & $.028^{\star}$ & .118 \\
\hline Valoración de la Situación como Desafío (VSD) & $.001^{\star \star}$ & $.005^{\star \star}$ & .730 \\
\hline Valoración de la Situación como Indiferente(VSI) & $.009^{\star *}$ & $.001^{* *}$ & $.008^{* *}$ \\
\hline $\begin{array}{l}\text { Afrontamiento Cognitivo dirigido a cambiar la } \\
\text { Situación (ACS) }\end{array}$ & .399 & $.001^{\star \star}$ & $.002^{\star *}$ \\
\hline $\begin{array}{l}\text { Afrontamiento Cognitivo dirigido a reducir la } \\
\text { Emoción (ACE) }\end{array}$ & $.041^{*}$ & $.028^{*}$ & .274 \\
\hline $\begin{array}{l}\text { Afrontamiento Conductual Motor dirigido a cambiar la } \\
\text { Situación (ACMS) }\end{array}$ & $.006^{\star \star}$ & $.011^{\star}$ & .523 \\
\hline $\begin{array}{l}\text { Afrontamiento Conductual Motor dirigido a reducir la } \\
\text { Emoción (ACME) }\end{array}$ & $.021^{*}$ & $.023^{*}$ & .313 \\
\hline Afrontamiento Pasivo (AP) & $.001^{\star \star}$ & .068 & .953 \\
\hline Afrontamiento Evitativo (AE) & $.001^{\star *}$ & $.001^{\star *}$ & .829 \\
\hline
\end{tabular}

${ }^{\star} p<.05 .{ }^{* *} p<.01$.

Actualidades en Psicología, 35(131), 2021, 1-18 
El contraste entre los voluntarios que han padecido una situación de estas características por fuera del rol de rescatistas de emergencias o catástrofe y aquellos que no lo han hecho, se evidenciaron diferencias en tres de las escalas analizadas: Valoración de la Situación como Amenazante $\left(p<.05 ; R_{1}\right.$ con experiencia $=88.11 ; R_{2}$ sin experiencia $=$ 107.72); Afrontamiento Pasivo $\left(p<.05 ; R_{1}\right.$ con experiencia $=89.69, R_{2}$ sin experiencia $=$ 106.58) y Afrontamiento Evitativo $\left(p<.01, R_{1}\right.$ con experiencia $=87.17, R_{2}$ sin experiencia $=$ 108.40). En todas las variables, el grupo sin experiencia como víctimas de situaciones de catástrofe presenta valores más elevados en estrategias poco productivas.

Eficacia en el programa de simulación de PAP

La eficacia en el entrenamiento es valorada a través de la cantidad de respuestas correctas, entendidas como aquellas que coinciden con las pautas de acción propuestas en el protocolo de PAP. El valor promedio de aciertos obtenidos en la muestra es de 6.03 con un DT de 1.84 (Mdn = 6; Mín. = 0; Máx. = 10). Resultados similares, referidos al desempeño en el programa de simulación, han sido presentados paralelamente en otros trabajos (Azzollini et al., 2017), contemplando una muestra más reducida, vinculando este desempeño a otras variables psicológicas analizadas en la muestra mencionada. En esta sección, se presentan los datos obtenidos en una muestra ampliada, para analizar posteriormente su vinculación con las estrategias de afrontamiento identificadas por los voluntarios.

En lo que respecta a la asociación de la eficacia en la aplicación del PAP y los datos demográficos, no se hallaron diferencias por género ni por tipo de institución, pero los valores de SIPAPSI muestran una relación significativa con la edad $\left(r_{s}=-.152 ; p<.05\right)$, lo que indicaría que las personas más jóvenes tienden a tener más éxito en la identificación de las conductas o actitudes correspondientes al protocolo de PAP propuesto.

La experiencia previa, ya sea como voluntario o como víctima de situaciones de catástrofe o emergencia, no mostró asociación con la cantidad de respuestas correctas en el protocolo simulado.

Estrategias de afrontamiento y rendimiento en el programa de simulación de PAP

El análisis de la asociación entre la eficacia en el simulador y las estrategias de afrontamiento señalan que existe una relación negativa entre la cantidad de respuestas correctas en SIPAPSI y los valores en las escalas del IVA. Esta relación resulta estadísticamente significativa en lo que respecta a la valoración de la situación como amenaza $\left(r_{s}=-.176 ; p<.05\right.$; esto es, que el individuo valora el evento como peligroso u hostil) o desafío $\left(r_{s}=-.168 ; p<.05\right.$; es decir, que el afrontamiento del estrés implica una respuesta de estrés frente a una situación novedosa, incierta, implicando el despliegue de estrategias poco aplicadas frente a situaciones previas) y no así en los distintos estilos de afrontamiento. La Tabla 4 muestra los valores obtenidos en toda la muestra.

Al dividir la muestra por género, las mujeres no presentan asociaciones significativas entre las respuestas correctas del SIPAPSI y los valores de las IVA, mientras que los varones muestran asociaciones entre las respuestas correctas en el SIPAPSI y el Afrontamiento Cognitivo dirigido a cambiar la Situación $\left(r_{s}=-.183 ; p<.05\right)$, el Afrontamiento Cognitivo dirigido a reducir la Emoción $\left(r_{s}=-.189 ; p<.05\right)$, Afrontamiento Conductual Motor dirigido a cambiar la Situación $\left(r_{s}=-.214 ; p<.05\right)$ y Afrontamiento Conductual Motor dirigido a reducir la Emoción $\left(r_{s}=-.265 ; p<.01\right)$. 
Tabla 4

Valores de correlación para los puntajes de la escala de IVA $(N=198)$

\begin{tabular}{lc}
\hline \multicolumn{1}{c}{ Escala IVA / Cantidad de respuestas correctas SIPAPSI } & $\begin{array}{c}\rho \text { de Spearman } \\
(\text { valor de } p)\end{array}$ \\
\hline Valoración de la Situación como Amenazante (VSA) & $-.176\left(.013^{\star}\right)$ \\
Valoración de la Situación como Desafío (VSD) & $-.168\left(.018^{\star}\right)$ \\
Valoración de la Situación como Indiferente(VSI) & $-.146(.400)$ \\
Afrontamiento Cognitivo dirigido a cambiar la Situación (ACS) & $-.124(.081)$ \\
Afrontamiento Cognitivo dirigido a reducir la Emoción (ACE) & $-.111(.120)$ \\
Afrontamiento Conductual Motor dirigido a cambiar la Situación (ACMS) & $-.133(.063)$ \\
Afrontamiento Conductual Motor dirigido a reducir la Emoción (ACME) & $-.098(.170)$ \\
Afrontamiento Pasivo (AP) & $-.060(.405)$ \\
Afrontamiento Evitativo (AE) & $-.110(.122)$ \\
\hline
\end{tabular}

Nota. $N=198$

${ }^{*} p<.05 .{ }^{* *} p<.01$.

Respecto a la correlación entre las respuestas en el programa de simulación con los indicadores de afrontamiento según el tipo de institución, se obtienen los resultados expresados en la Tabla 5.

Las estrategias de afrontamiento guardan relación con la eficacia en PAP en el grupo de Bomberos Voluntarios, pero no en los otros dos grupos. Se observan asociaciones entre 6 escalas del IVA y la cantidad de respuestas correctas en la simulación: Valoración de la Situación como Amenazante $\left(r_{s}=-.197\right)$; Valoración de la Situación como Desafío $\left(r_{s}=-.200 ; p<.05\right)$; Valoración de la Situación como Indiferente $\left(r_{s}=-.249 ; p<.05\right)$; Afrontamiento Cognitivo dirigido a cambiar la Situación $\left(r_{s}=-.202 ; p<.05\right)$; Afrontamiento Conductual Motor dirigido a cambiar la Situación $\left(r_{s}=-.199 ; p<.05\right)$; Afrontamiento Evitativo $\left(r_{s}=-.299 ; p<.05\right)$.

Los sujetos que han tenido experiencia previa como voluntarios muestran asociaciones entre la cantidad de respuestas correctas del SIPAPSI y la escala Valoración de la Situación como Indiferente $\left(r_{s}=-.215 ; p<.05\right)$, sin presentar otras covariaciones entre las escalas evaluadas. En los voluntarios sin experiencia previa, la eficacia en el entrenamiento muestra mayor cantidad de asociaciones con las estrategias de afrontamiento: Valoración de la Situación como Amenazante $\left(r_{s}=-.254 ; p<.05\right)$, Valoración de la Situación como Desafío $\left(r_{s}=-.274 ; p<.01\right)$ y Afrontamiento Conductual Motor dirigido a reducir la Emoción $(r$ $=-.227 ; p<.05)$. En este grupo, las tendencias indican que se identifican con un número menor de las estrategias mencionadas y que tienden a presentar mejor eficacia en la aplicación de PAP.

Al analizar la covariación entre los puntajes obtenidos en el SIPAPSI y los resultados de la escala según la experiencia previa de los voluntarios como víctimas por fuera de su rol de rescatistas, se observa que quienes han padecido situaciones de dichas características muestran una leve tendencia a presentar mejor rendimiento en la simulación, cuanto menos perciben a la situación como indiferente $\left(r_{s}=-.248 ; p<.05\right)$. En quienes no han atravesado este tipo de situaciones, la eficacia en el programa SIPAPSI se encuentra modulada por un mayor número estrategias de afrontamiento que presenta asociaciones entre las respuestas correctas alcanzadas y las siguientes escalas: Valoración de la 
Tabla 5

Valores de correlación para los puntajes de la escala IVA con el número de respuestas correctas

\begin{tabular}{|c|c|c|c|}
\hline \multirow[b]{2}{*}{$\begin{array}{l}\text { Escala IVA / Cantidad de respuestas correctas } \\
\qquad \text { SIPAPSI }\end{array}$} & \multicolumn{3}{|c|}{$\rho$ de Spearman (Valor de $p$ ) } \\
\hline & $\begin{array}{c}\text { Bomberos } \\
\text { Voluntarios } \\
(n=139)\end{array}$ & $\begin{array}{c}\text { Cruz } \\
\text { Roja } \\
(n=42)\end{array}$ & $\begin{array}{c}\text { Defensa } \\
\text { Civil } \\
(n=17)\end{array}$ \\
\hline Valoración de la Situación como Amenazante (VSA) & $-.197(.019 *)$ & $-.351(.022)$ & $-.076(.770)$ \\
\hline Valoración de la Situación como Desafío (VSD) & $-.200\left(.018^{\star}\right)$ & $-.303(.050)$ & $-.265(.303)$ \\
\hline Valoración de la Situación como Indiferente(VSI) & $-.249\left(.003^{\star \star}\right)$ & $-.036(.817)$ & $.171(.509)$ \\
\hline $\begin{array}{l}\text { Afrontamiento Cognitivo dirigido a cambiar la } \\
\text { Situación (ACS) }\end{array}$ & $-.202\left(.016^{\star}\right)$ & $.092(.560)$ & $-.035(.891)$ \\
\hline $\begin{array}{l}\text { Afrontamiento Cognitivo dirigido a reducir la } \\
\text { Emoción (ACE) }\end{array}$ & $-.152(.073)$ & $.024(.878)$ & $-.137(.598)$ \\
\hline $\begin{array}{l}\text { Afrontamiento Conductual Motor dirigido a } \\
\text { cambiar la Situación (ACMS) }\end{array}$ & $-.199\left(.018^{\star}\right)$ & $.138(.380)$ & $-.380(.131)$ \\
\hline $\begin{array}{l}\text { Afrontamiento Conductual Motor dirigido a } \\
\text { reducir la Emoción (ACME) }\end{array}$ & $-.116(.170)$ & $-.098(.534)$ & $-.344(.175)$ \\
\hline Afrontamiento Pasivo (AP) & $-.173(.041)$ & $.010(.948)$ & $.272(.289)$ \\
\hline Afrontamiento Evitativo (AE) & $-.229\left(.006^{\star \star}\right)$ & $-.141(.370)$ & $.216(.402)$ \\
\hline
\end{tabular}

${ }^{*} p<.05 .{ }^{* *} p<.01$.

Situación como Amenazante $\left(r_{s}=.264 ; p<.01\right)$, Valoración de la Situación como Desafío $\left(r_{s}=-.248 ; p<.01\right)$ y Afrontamiento Conductual Motor dirigido a reducir la Emoción $\left(r_{s}\right.$ $=-.217 ; p<.05$ ). Las escalas que modulan la eficacia en el programa de simulación en este grupo son las mismas que modulan la eficacia del entrenamiento en el grupo sin experiencia como voluntarios.

\section{Discusión}

El análisis estadístico realizado en la presente investigación buscaba determinar la eficacia en el programa de simulación y entrenamiento en Primera Ayuda Psicológica (PAP) en voluntarios de diferentes instituciones y la relación de su desempeño con el estilo de afrontamiento.

Los resultados hallados indican que el rendimiento en el programa de simulación resultó satisfactorio en los tres grupos evaluados, lo cual se considera como un indicador favorable respecto al posible desarrollo de habilidades para la identificación de las conductas propuestas en la capacitación. Este supuesto se halla en línea con lo citado en la introducción del trabajo en el que varios autores (Crawford et al., 2015; Reifels et al., 2014) plantean que el hecho de proporcionar capacitación en PAP provocaría también mayor propensión a aplicar dichas habilidades a la comunidad en general, ya que los rescatistas son una parte importante de la fuerza de trabajo en las situaciones de emergencia. El uso del programa de simulación, para el entrenamiento y evaluación del personal de Bomberos Voluntarios, Defensa Civil y Cruz Roja concuerda con Kureckova et al. (2017) respecto a que el trabajo en este tipo de población se orienta a analizar el desempeño, la eficacia y los procesos emocionales desplegados mediante simulación para que ayudaran a manejar las situaciones de emergencia de manera efectiva y así disminuir las reacciones emocionales inadecuadas. 
Las características de afrontamiento de los voluntarios se asociaron levemente a la cantidad de respuestas correctas en el SIPAPSI, hallándose diferencias entre los subgrupos de acuerdo con su género, edad, institución y experiencia previa.

Al resumir los hallazgos en cada una de las dimensiones analizadas, puede afirmarse que en cuanto a las estrategias de afrontamiento, al tomar la muestra en su conjunto, se observó que predomina la Valoración de la Situación como Desafío y las estrategias de afrontamiento activas, cognitivas y motoras dirigidas a disminuir la emoción. Esta característica es concordante con el protocolo de PAP propuesto, en el que se busca disminuir el efecto de las emociones disparadas por la situación de estrés a través de la promoción de la activación cognitiva (Farchi et al., 2014). Las estrategias de afrontamiento presentaban diferencias menores al mostrar que los bomberos voluntarios, las mujeres mayores, y los voluntarios con experiencia previa, tienen mayor cantidad de respuestas acertadas en la simulación.

La prevalencia de estrategias activas de afrontamiento resulta según las evidencias halladas, que son relevantes en la conformación de un perfil efectivo de voluntarios. Los resultados alientan a formular la hipótesis de que la valoración de las situaciones de emergencia o catástrofe en términos de desafío, caracterizarían a los voluntarios de distintas instituciones, lo cual permite aprender y aplicar con mayor efectividad los protocolos de acción de PAP. La consideración de estos factores en el reclutamiento y capacitación de voluntarios resultaría un aporte relevante. Como resultado se presentarían características deseables en aquellos que deben asistir a víctimas en contextos de peligro y desorganización como se configuran las emergencias y desastres.

La implementación de estrategias activas orientadas a la disminución de la emoción y a la modificación de la situación, también son valoradas a la luz de los resultados y en términos favorables, para la asistencia de personas en contextos de gran complejidad. Un afrontamiento activo parece proteger la salud de los voluntarios a la vez que promueve una asistencia eficaz a las víctimas. En línea con dichos hallazgos, y la idea concluyente de que la disminución de la emoción mejoraría la aplicación de la PAP, estudios recientes realizados que aplican nuevamente el software mencionado, mostraron que los voluntarios que portaban un estilo de toma de decisiones de tipo urgente "afectivo" dotado de componentes emocionales (Cosentino et al., 2017) y que a su vez habían respondido a la tarea de aplicación del Modelo de las "4 C" mediante una consigna también de tipo emocional ("decida según lo que sienta emocionalmente"; Betsch \& Kunz, 2008; Depaula, 2016; Depaula \& Azzollini, 2019) mostraron un rendimiento menos efectivo o inferior en comparación a los sujetos movilizados durante la prueba por un estilo y consigna experimental de tipo racional o deliberada. Incluso, los autores no hallaron diferencias en el rendimiento al operar el SIPAPSI entre los sujetos más "emocionales" y un grupo control que no había recibido la capacitación en PAP previamente (Azzollini et al., 2018; Grinhauz et al., 2020).

Para finalizar, estudios posteriores, permitirán ampliar el análisis de características de personalidad deseables en rescatistas de distintas instituciones, que favorezcan el aprendizaje y la ejecución de PAP en situaciones de emergencia.

\section{Referencias}

Ascaini, I., Luna, M. E., \& Tejo, M. (2016). Intervenciones psico-sociales en catástrofes socio-naturales. Experiencias extensionistas de la facultad de psicología de la UNLP. Anuario Temas en Psicología, 1, 51-73.

Actualidades en Psicología, 35(131), 2021, 1-18 
Azzara, S., Grinhauz, A. S., \& Azzollini, S. (2019). Acciones de transferencia en primera ayuda psicológica a organizaciones involucradas en acciones de rescate en situaciones de crisis: Relación entre la efectividad y la tendencia individual decisoria. Revista Argentina de Ciencias del Comportamiento, 11(2), 1-9.

Azzollini, S. C., Cosentino, A. C., Depaula, P. D., Becerra, L., \& Porte Petit Ortiz, A. (2015). SIPAPSI: Sistema Interactivo de Primera Ayuda Psicológica (Versión 1).

Azzollini, S. C., Depaula, P. D., Cosentino, A. C., \& Bail, V. P. (2018). Applications of Psychological First Aid in Disaster and Emergency Situations: Its Relationship with Decision-Making. Athens Journal of Social Sciences, 5(2), 201-213. https://doi. org/10.30958/ajss_v5i2

Azzollini, S., Azzara, S., Depaula, P., Cosentino, A., \& Grinhauz, A. S. (2017). Modificación del Protocolo de Primera Ayuda Psicológica (PAP) en Situaciones de Crisis Basado en el Modelo de las 4 C. Acta Psiquiátrica y Psicológica de América Latina, 63(2), 139-147.

Bail, V. P., Becerra, L., Azzollini, S. C., Depaula, P., \& Cosentino, A. C. (2017). Perfil de voluntarios para la asistencia en emergencias y catástrofes: La empatía y el entrenamiento en Primera Atención Psicológica [Memoria]. Memorias IX Congreso Internacional de Investigaciones y Práctica Profesional en Psicología, XXIV Jornadas de Investigación, XIII Encuentro de Investigaciones en Psicología del MERCOSUR. Ediciones de la Facultad.

Betsch, C., \& Kunz, J. J. (2008). Individual Strategy Preferences and Decisional Fit. Journal of Behavioral Decision Making, 21, 532-555. https://doi.org/10.1002/bdm.600

Biggs, Q. M., Fullerton, C. S., McCarroll, J. E., Liu, X., Wang, L., Dacuyan, N. M., Zatzick, D. F., \& Ursano, R. J. (2016). Early intervention for post-traumatic stress disorder, depression, and quality of life in mortuary affairs soldiers postdeployment. Military Medicine, 187(11-12), e1553-e1560. https://doi.org/10.7205/MILMED-D-15-00579

Brooks, S. K., Dunn, R., Amlôt, R., Greenberg, N., \& Rubin, G. J. (2016). Social and occupational factors associated with psychological distress and disorder among disaster responders: a systematic review. BMC Psychology, 18(4), 1-13. https://doi. org/10.1186/s40359-016-0120-9

Cano-Vindel, A., \& Miguel-Tobal, J. J. (1999). Evaluación de la ansiedad desde un enfoque interactivo y multidimensional: El inventario de Situaciones y Respuestas de Ansiedad (ISRA). Psicología Contemporánea, 6(1), 14-21.

Colussi, M. (2017). Trabajo psicológico en situaciones de desastres. Revista Análisis de la Realidad Nacional, 6(117), 129-152.

Cosentino, A. C., Azzollini, S. C., Depaula, P. D., \& Castillo, S. E. (2017). Assessment of the Affective and Rational Bases for Urgent Decision-Making under Extreme Circumstances. Análise Psicológica, 35(4), 529-542. https://doi.org/10.14417/ap.1267

Crawford, G., Burns, S. K., Chih, H. J., Hunt, K., Tilley, P. J., Hallett, J., Coleman, K., \& Smith, S. (2015). Mental health first aid training for nursing students: a protocol for a pragmatic randomized controlled trial in a large university. BMC Psychiatry, 26(15), 1-8. https://doi.org/10.1186/s12888-015-0403-3

De Leo, G., Ponder, M., Molet, T., Fato, M., Thalmann, D., Magnenat-Thalmann, N., Bermano, F., \& Beltrame, F. (2003). A Virtual Reality System for the Training of Volunteers 
Involved in Health Emergency Situations. Cyber Psychology \& Behavior, 6(3), 267274. https://doi.org/10.1089/109493103322011551

Depaula, P. D. (2016). Toma de decisiones en ambientes culturales diversos. [Tesis doctoral no publicada], Universidad de Buenos Aires.

Depaula, P. D., \& Azzollini, S. C. (2019). Predictores culturales de la efectividad decisoria: Estudio experimental con cascos azules argentinos. Revista de Psicología, 37(1), 53-97. https://doi.org/10.18800/psico.201901.003

Dieltjens, T., Moonens, I., Van Praet, K., De Buck, E., \& Vandekerckhove, P. (2014). A Systematic Literature search on Psychological First Aid: Lack of Evidence to Develop Guidelines. PLoS ONE, 9(12). https://doi.org/10.1371/journal.pone.0114714

El Proyecto Esfera. (2011). Carta Humanitaria y normas mínimas para la respuesta humanitaria (3 $\quad$ Ed.). https://www.acnur.org/fileadmin/Documentos/ Publicaciones/2011/8206.pdf

Everly, G. S., \& Flynn, B. W. (2005). Principles and practice of acute psychological first aid after disasters. In G. S. Everly., \& C. L. Parker (Eds.), Mental health aspects of disasters: Public health preparedness and response, revised (pp. 79-89). Johns Hopkins Center for Public Health Preparedness.

Farchi, M., Cohen, A., \& Mosek, A. (2014). Developing Specific Self-Efficacy and Resilience as First Responders among Students of Social Work and Stress and Trauma Studies. Journal of Teaching in Social Work, 34, 129-146. https://doi.org/10.1080 /08841233.2014.894602

Farchi, M., \& Hantman, S. (2013). From Helplessness to active coping. En E. Schott., \& E. Weiss (Eds.). Social Work Practice in Health, Mental Health \& Communities: A Meta-framework for Micro, Mezzo, Macro and Global Action. USC School of Social Work Department of Military Social Work.

Farchi, M., Levy, B. T., Gershon, B. B., Hirsch-Gornemann, B. M., Whiteson, A., \& Gidron, Y. (2018). The SIX model for Immediate Cognitive Psychological First Aid: From Helplessness to Active Efficient Coping. International Journal Of Emergency Mental Health and Human Resilience, 20(2), 1-12. https://doi.org/10.4172/1522-4821.1000395

Grinhauz, A., Azzara, S. H., Otamendi, A. M., \& Azzollini, S. (2020). La toma de decisiones de rescatistas y la efectividad en la primera ayuda psicológica. Interdisciplinaria, 7(2), 23-37. https://doi.org/10.16888/interd.2020.37.2.2

Ha, K. M., Park, S., Yoon, Y., Nam, K. H., \& Oh, H. M. (2015). Emergency management training in Korea: combining and balancing supply- and demand-centered paradigms. Springer Plus, 653(4), 1-8. https://doi.org/10.1186/s40064-0151459-8

Hambrick, E. P., Rubens, S. L., Vernberg, E. M., Jacobs, A. K., \& Kanine, R. M. (2014). Towards Successful Dissemination of Psychological First Aid: A Study of Provider Training Preferences. The Journal of Behavioral Health Services \& Research, 41, 203-215. https://doi.org/10.1007/s11414-013-9362-y

Herrman, H. (2012). Promoting Mental Health and Resilience after a Disaster. Journal of Experimental and Clinical Medicine, 4(2), 82-87. https://doi.org/10.1016/j. jecm.2012.01.003 
Hogarth, R. M., \& Soyer, E. (2015). Providing information for decision making: Contrasting description and simulation. Journal of Applied Research in Memory and Cognition, 4, 221-228. https://doi.org/10.1016/j.jarmac.2014.01.005

Inter-Agency Standing Committee. (2007). IASC Guidelines on Mental Health and Psychosocial Support in Emergency Settings.

Kearns, M. C., Ressler, K. J., Zatzick D., \& Rothbaum, B. O. (2012). Early interventions for PTSD: a review. Depression and Anxiety, 29(10), 833-842. https://doi.org/10.1002/ da.21997

Kureckova, V., Gabrhel, V., Zamecnik, P., Rezac, P., Zaoral, A., \& Hobl, J. (2017). First aid as an important traffic safety factor-evaluation of the experience-based training. European Transport Research Review, 9(5), 1-8. https://doi.org/10.1007/s12544016-0218-4

Leibovich de Figueroa, N. B. (1999). Adaptación del Inventario de Valoración y Afrontamiento (IVA). Departamento de Publicaciones, Facultad de Psicología.

Levy, E., Farchi, M., Gidron, Y., \& Shahar, E. (2020). Psychological first aid through the 'SIX Cs model' - an intervention with migrants on the move. Intervention, 18(1), 71-77. https://doi.org/ 10.4103/INTV.INTV_51_18

McCabe, O. L., Everly Jr., G. S., Brown, L. M., Wendelboe, A. M., Abd Hamid, N. H., Tallchief, V. L., Links, J. (2014). Psychological First Aid: A Consensus-Derived, Empirically Supported, Competency-Based Training Model. American Journal of Public Health, 104(4), 621-628. https://doi.org/10.2105/AJPH.2013.301219

National Child Traumatic Stress Network \& National Center for PTSD. (2006). Psychological first aid: field operations guide. https://www.ptsd.va.gov/professional/treat/type/ PFA/PFA_2ndEditionwithappendices.pdf

Navarro, J. (2004). Enfermedad y familia: manual de intervención psicosocial. Grupo Planeta.

Organización Mundial de la Salud, War Trauma Foundation, \& Visión Mundial Internacional. (2012). Primera ayuda psicológica: Guía para trabajadores de campo.

Palacios, B., \& Téllez, M. B. (2017). La importancia de la atención a la salud mental en una situación de desastre. Experiencia de brigadas de apoyo psicológico de emergencia universitarias después del Sismo del 19 de septiembre 2017 en México. Revista Salud UIS, 49(4), 513-516.

Plummer, C. A., Cain, D. S., Fisher, R. M., \& Bankston, T. Q. (2009). Practice Challenges in Using Psychological First Aid in a Group Format with Children: A Pilot Study. Brief Treatment and Crisis Intervention, 8, 313-326. https://doi.org/10.1093/brieftreatment/mhn019

Qi, W., Gevonden, M., \& Shalev, A. (2016). Prevention of post-traumatic stress disorder after trauma: current evidence and future directions. Current Psychiatry Reports, 18(2), 20. https://doi.org/10.1007/s11920-015-0655-0

Reifels, L., Naccarella, L., Blashki, G., \& Pirkis, J. (2014). Examining Disaster Mental Health Workforce Capacity. Psychiatry, 77(2), 199-205. https://doi.org/10.1521/ psyc.2014.77.2.199 
Roberts, N. P., Kitchiner, N. J., Kenardy, J., Robertson, L., Lewis, C., \& Bisson, J. I. (2019). Multiple session early psychological interventions for the prevention of posttraumatic stress disorder. Cochrane Database of Systematic Reviews, 8(CD006869), 1-129. https://doi.org/10.1002/14651858.CD006869.pub3

Sun, J. H., \& Wallis, L. H. (2011). The psychological effects of widespread emergencies and a first responder training course on a violent, developing community. African Journal of Emergency Medicine, 1, 166-173. https://doi.org/10.1016/j. afjem.2011.10.007

Svetlitzky, V., Farchi, M., Ben Yehuda, A., \& Adler, A. B. (2019). YaHaLOM: A Rapid Intervention for Acute Stress Reactions in High-Risk Occupations. Military Behavioral Health, 232-242. https://doi.org/10.1080/21635781.2019.1664356

Wijesinghe, C. A., Williams, S. S., Kasturiratne, A., Dolawaththa N., Wimalaratne, P., Wijewickrema, B., Jayamanne, S., Isbister, G., Dawson, A., Lallo, D., Janaka de Silva, H. (2015). A randomized controlled trial of a brief intervention for delayed psychological effects in snakebite victims. PLoS Neglected Tropical Diseases, 9(8), e0003989. https://doi.org/10.1371/journal.pntd.0003989 